\title{
Prevalencia y factores relacionados con el consumo de alcohol en adolescentes embarazadas: una revisión sistemática
}

\author{
Roger Quintana-Lagunas', Nora Angélica Armendáriz-García', Luis Iván Mayor Silva² \\ I Facultad de Enfermería. Universidad Autónoma de Nuevo León \\ 2 Facultad de Enfermería, Fisioterapia y Podología. Universidad Complutense de Madrid
}

\section{RESUMEN}

Introducción: el consumo de alcohol durante el embarazo (CADE) produce problemas físicos, cognitivos y de comportamiento en la madre y en el bebé en gestación, por ello se recomienda la abstinencia de esta sustancia durante el embarazo; sin embargo, factores presentes en los adolescentes podrían relacionarse con el inicio o continuación del consumo de alcohol mientras se está embarazada. Objetivo: identificar las prevalencias y factores relacionados con el consumo de alcohol en adolescentes embarazadas (AE) por medio de una revisión sistemática. Método: búsqueda de artículos en inglés y español entre 2009 y el 2019 en AE. Las bases de datos fueron PubMed, Web of Science: Social Sciences Citation Index, Science Citation Index y Scopus. Los términos de búsqueda incluyeron las palabras "pregnancy", "pregnant", "adolescent", "adolescence" y "alcohol". La extracción de los datos fue independiente por un autor utilizando los indicadores de calidad del estudio; se determinaron prevalencias del CADE en AE y factores personales, psicológicos y espirituales relacionados. Resultados: el consumo de alcohol previo al embarazo y la presencia de embarazos anteriores fueron los más mencionados como factores personales. Asimismo, se identificaron relaciones de factores psicológicos como depresión, ansiedad, factores sociales como solo tener un padre, tener amigos que fuman o toman, así como no contar con pareja. Por último, se encontraron algunos factores espirituales entre los que destaca la importancia brindada a la religión. Conclusiones: el consumo de alcohol es multifactorial, sin embargo, existen factores personales, psicológicos, sociales y espirituales que aumentan el riesgo hasta cuatros veces más de consumir alcohol.

Palabras clave: prevalencia, factores, embarazo, adolescente, alcohol.

\begin{abstract}
Introduction: alcohol consumption during pregnancy causes physical, cognitive and behavioral problems in the mother and the unborn baby. The literature recommends abstinence from this substance during pregnancy. However, the presence of factors in adolescents could be related to the initiation or continuation of alcohol consumption while pregnant. Objective: twas to identify the prevalence and factors that are related to alcohol consumption in pregnant adolescents (AE). Method: a systematic review of articles in English and Spanish published between 2009 and 2019 in the adolescent population was carried out. The databases were PubMed, Web of Science: Social Sciences Citation Index, Science Citation Index, and Scopus. The search terms included the words "pregnancy," "pregnant," "adolescent," "adolescence," and "alcohol." Data extraction was performed independently by one author using the study quality indicators. Results: CADE prevalence's in AE were determined. Also, personal, psychological and spiritual factors related to CADE. Alcohol consumption prior to pregnancy and exposure to the presence of previous pregnancies were the most mentioned as personal factors. Likewise, relationships of psychological factors such as depression and anxiety were identified. In addition, social factors were identified such as having a single parent, having friends who smoke or drink, as well as not having a partner. Finally, some spiritual factors were found, among which the importance given to religion stands out. Conclusions: alcohol consumption during pregnancy is multifactorial, however, there are personal, psychological, social and spiritual factors that increase the risk of consuming alcohol up to four times more.
\end{abstract}

Keywords: prevalence, factors, pregnancy, adolescent, alcohol.

\footnotetext{
Autor de correspondencia:

Nora Angélica Armendáriz-García, Facultad de Enfermería, Universidad Autónoma de Nuevo León, Monterrey

Correo electrónico:nora.armendarizg@uanl.mx

Recibido: 12 de enero de 2021

Aceptado: 18 de marzo de 2021

doi: 10.28931/riiad.2021.1.06
} 


\section{INTRODUCCIÓN}

\section{Factores relacionados con el consumo de alcohol en adolescentes embarazadas}

El consumo de alcohol durante el embarazo (CADE) produce un nivel de toxicidad en la madre que afecta el desarrollo pre y posnatal del producto (Popova et al., 2017). Una de cada 67 mujeres embarazadas que consume alcohol dará a luz a un niño con Trastorno Espectral Alcohólico Fetal (TEAF) cada año en todo el mundo, el cual se caracteriza por problemas físicos, cognitivos y de comportamiento en niños de madres bebedoras (Ruisch et al., 2017). Está ampliamente comprobado que no hay cantidad o tipo de alcohol que se considere seguro de ingerir durante el embarazo y que es indispensable su abstinencia durante todo el periodo de gestación (Lange et al., 2017; Organización Mundial de la Salud [OMS], 2019; Popova et al., 2017). No obstante, las prevalencias de consumo de alcohol durante el embarazo en diversos países de Europa y América son alarmantes (Irlanda, 60\%; Belarús, 47\%; Brasil, 15.2\% y México 1.2\%; Bottorff et al., 2014; Lange et al., 2017; Onwuka et al., 2016).

Estudios acerca del CADE en mujeres adultas han mostrado algunas prevalencias (Lange et al., 2017) y relaciones entre diversos factores presentes, tales como la edad, el estado civil, el nivel de escolaridad, la depresión y la permisividad por parte de los padres y/o de la pareja (Bottorff et al., 2014; Skagerström et al., 2013). Sin embargo, los resultados no han sido concluyentes en población adolescente. No se han identificado revisiones sistemáticas recientes en esta población y aquellas que existen no abordan el fenómeno del consumo de alcohol durante el embarazo desde un enfoque holístico, que incluya diversos factores como los personales, psicológicos, sociales y espirituales debido a que la problemática del consumo de alcohol no es unifactorial, sino que está compuesta por diversos factores. Se considera que las adolescentes embarazadas sufren cambios en todos los aspectos de su vida, por tal motivo, se espera que esta revisión sistemática ayude a comprender que factores personales, psicológicos, sociales y espirituales se relacionan con el consumo de alcohol. Los resultados podrían mejorar los esfuerzos en la atención prenatal en el embarazo adolescente para la abstinencia de alcohol en esta etapa para redoblar esfuerzos en la prevención del consumo de alcohol en embarazadas y reducir las tasas de los trastornos del espectro alcohólico fetal (Lange et al., 2017).

\section{MÉTODO}

La presente revisión sistemática consideró los ítems de referencia para publicar protocolos de revisiones sis- temáticas y meta-análisis (PRISMA, por sus siglas en inglés). Los métodos de análisis y los criterios de inclusión se especificaron a priori y se documentaron en un protocolo que se encuentra disponible en el correo de correspondencia.

\section{Criterios de elegibilidad}

Características del estudio: Se aplicaron restricciones de fecha de publicación a partir del 2009, asimismo, se impusieron restricciones de idioma en inglés y español; además, sólo se incluyeron estudios originales de casos y controles, estudios de cohorte, estudios transversales con prevalencias y estudios originales que abordaron uno o varios de los factores personales, psicológicos, sociales y espirituales relacionados con el consumo de alcohol en adolescentes embarazadas. Se identificó y analizó la evidencia publicada entre los años 2009 y 2019 en población adolescente; los factores son aquellos elementos como la edad, depresión, familia o religión que pueden condicionar el embarazo y contribuir o ser determinantes para que se consuma alcohol durante el mismo y que en conjunto condicionan la salud. Se revisaron estudios primarios publicados de diseño descriptivo correlacional.

Características de los participantes: mujeres embarazadas de entre 12 y 19 años de edad. Los datos de mujeres de 20 años o más se excluyeron de esta revisión, ya que se encuentran fuera del rango considerado en la etapa de la adolescencia.

Tipos de medidas de resultado: prevalencias de CADE, así como la presencia, ausencia y tamaño de relación o efecto de los factores personales, psicológicos, sociales, culturales y espirituales con el CADE.

\section{Fuentes de información}

Los estudios se identificaron indagando en las bases de datos electrónicas, al revisar la lista de referencia de artículos y consultando con expertos en el tema. La búsqueda se realizó en PubMed, Web of Science en Social Sciences Citation Index y Science Citation Index, Scopus y Pro Quest, durante los meses de octubre y noviembre de 2019, la última se efectuó el 30 de noviembre de 2019.

\section{Búsqueda}

Se utilizaron los siguientes términos para buscar en todos los registros de las bases de datos. A continuación, se presenta la estrategia de búsqueda utilizada en la base de datos de PubMed.

((pregnan*[Title]) AND adolescen*[Title]) AND alcohol[Title] 


\section{Selección de los estudios}

La evaluación de la elegibilidad fue realizada de manera independiente por un revisor, quien examinó los registros a nivel de título y resumen utilizando los criterios de inclusión mencionados anteriormente, se clasificaron en tres grupos "incluir", "excluir" e "inseguro". Los clasificados bajo "incluir" o "inseguro" se examinaron en una segunda ronda a texto completo y con base en los resultados, los artículos de texto completo fueron evaluados de forma independiente con lo que se crearon tablas que utilizan criterios de inclusión de selección más detallados conforme a los criterios de elegibilidad. En una tercera ronda, los registros se clasificaron en dos grupos "inclusión" o "exclusión".

La cantidad total de estudios identificados a partir de las bases de datos, los seleccionados según los criterios de elegibilidad, los estudios finales incluidos en la revisión, y los que fueron excluidos con sus respectivos motivos se encuentran en un diagrama de flujo recomendado por las directrices PRISMA. Durante el proceso de selección de registros se utilizó la herramienta Mendeley para exportar, organizar y eliminar duplicados de los registros.

\section{Proceso de recopilación de datos}

Se desarrolló una hoja de extracción de datos basada en los objetivos de la revisión y refinada en consecuencia; se extrajeron los datos de los estudios incluidos y al final se hizo una evaluación por parte de un segundo revisor.

\section{Riesgo de sesgo en los estudios individuales}

Para determinar la validez de los estudios primarios elegibles, el investigador principal trabajó de forma independiente con la finalidad de determinar la idoneidad de la metodología utilizada en los estudios observacionales mediante la utilización de la lista de verificación de las directrices para la comunicación de estudios observacionales (STROBE, por sus siglas en inglés). Esta declaración está compuesta por 22 puntos que se consideran esenciales para una comunicación adecuada de los estudios observacionales.

\section{Análisis de datos}

Se extrajo la información de cada registro sobre características de los participantes, las prevalencias del CADE y los factores personales, psicológicos, sociales y espirituales.

\section{Medidas de resumen}

La medida de resultado primaria para el análisis cuantitativo fue la prevalencia de consumo de alcohol durante el embarazo en adolescentes y la probabilidad de ocurrencia (OR) con intervalos de confianza de 95\%.

\section{RESULTADOS}

La búsqueda en las bases de datos PubMed, Web of Science en Social Sciences Citation Index, Science Citation Index, Scopus y por medio de otras fuentes proporcionó un total de 50 registros. Después de eliminar los duplicados restaron 32 estudios, de los cuales 20 fueron descartados debido a que después de revisar los títulos y resúmenes se encontró que estos artículos no cumplían con los criterios claramente. Ocho estudios adicionales se excluyeron porque al leer el texto completo no cumplían con los criterios. cuatro estudios cumplieron con los criterios de inclusión y se incluyeron en análisis (Ver diagrama de flujo, Figura 1).

\section{Descripción del diagrama de flujo}

Se compararon los títulos de los 64 estudios potenciales con los criterios de inclusión para determinar cuáles artículos eran elegibles para la inclusión; este proceso de selección produjo 32 artículos potencialmente elegibles. Los primeros fueron obtenidos y leídos a título y resumen, este proceso identificó 12 artículos potenciales y se excluyeron 20 que no cumplieron con los criterios de fecha de publicación, entre otros criterios.

Después de una revisión a texto completo se eliminaron cuatro de ellos por no abordar ninguna variable respecto al consumo y cuatro no cumplieron con los criterios de inclusión por edad de las embarazadas. El número final de estudios incluidos en la revisión sistemática fue de cuatro (Figura 1).

\section{Características de los estudios incluidos}

De los cuatro estudios finalmente seleccionados para la revisión, dos fueron transversales y dos longitudinales, tres fueron en inglés y uno en español; la recolección de datos en dos de los artículos fue por medio de cuestionarios auto-aplicados dos por medio de fuentes secundarias. Todos los estudios incluyeron la asignación del muestreo, las participantes de los estudios incluidos fueron mujeres embarazadas de entre 12 y 19 años de edad, a excepción de dos estudios que abarcaron grupos de embarazadas de hasta 20 años, sin embargo, se aseguró que en el grupo se encontraran también mujeres con 19 años o menos. 


\section{Figura 1.}

Diagrama de flujo de la selección de los estudios

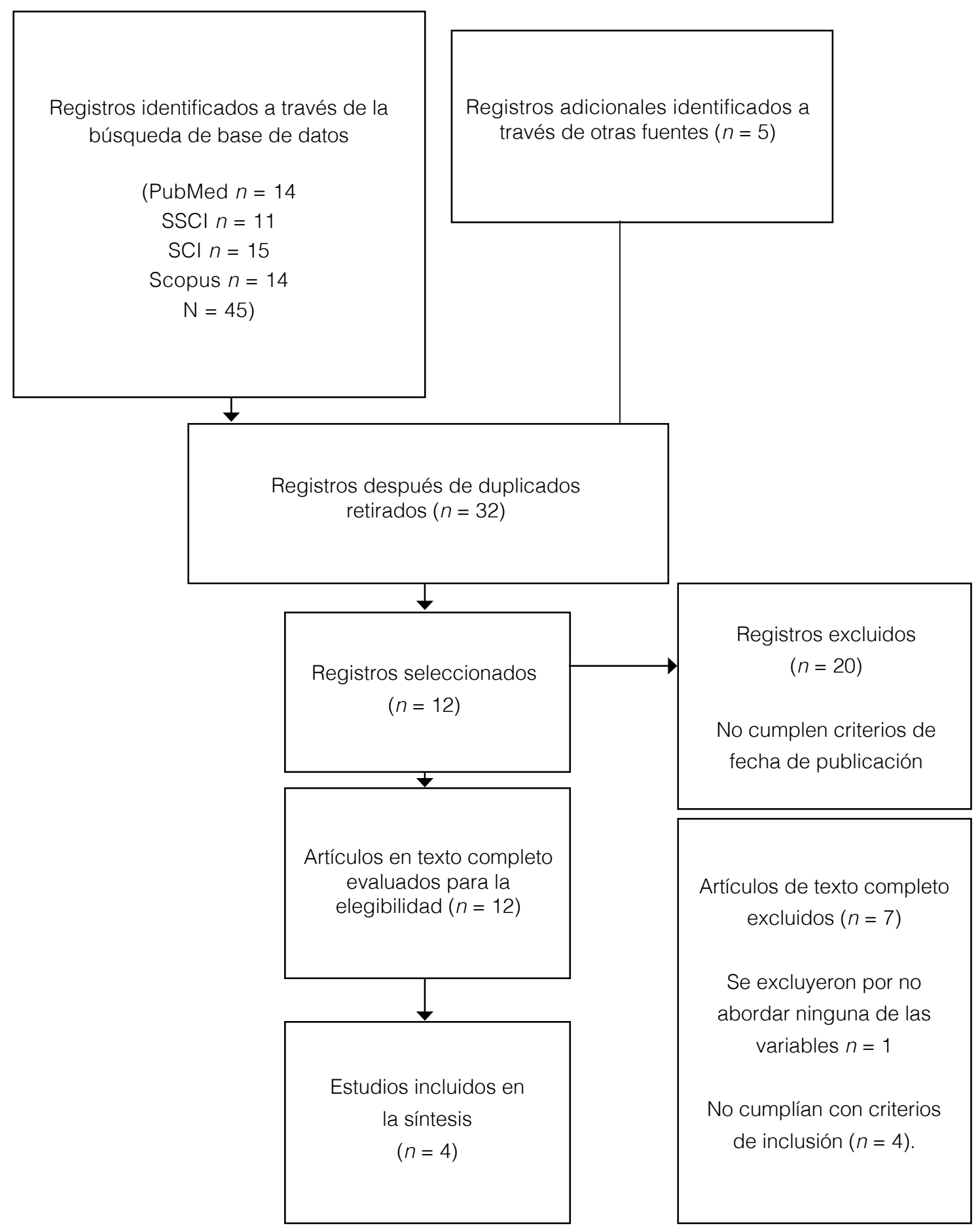


Realización de los estudios: dos fueron realizados en un ambiente de atención prenatal y dos fueron aplicados vía telefónica. Resultados primarios: en todos los estudios el resultado primario fue el consumo de alcohol durante el embarazo, medido a través de las prevalencias del uso.

\section{Resultados secundarios y adicionales}

Se extrajeron los riesgos relativos de las principales características de las adolescentes que consumieron esta sustancia (factores personales, psicológicos, sociales, espirituales). Además, uno de los estudios incluidos evaluó factores protectores presentes en aquellas adolescentes que no consumieron alcohol (importancia de la religión).

\section{Síntesis de los resultados}

La prevalencia de CADE entre adolescentes se presentó entre $15.8 \%$ y $41.5 \%$ (Tabla 1). Los datos de factores relacionados con el consumo de alcohol en adolescentes embarazadas estuvieron disponibles en la totalidad de los estudios incluidos, los datos agrupados se basaron en factores personales $(n=2)$, psicológicos $(n=2)$, sociales $(n=2)$ y espirituales (1). Respecto a los factores personales (Tabla 2) se observó heterogeneidad entre los estudios en cuanto a los factores personales principalmente, identificando que embarazos previos y consumo previo al embarazo se relacionan con el consumo durante esta etapa (Salas-Wright et al., 2015; Veloso \& Monteiro, 2013). Asimismo, se identificó que tener entre 15 y 17 años de edad aumenta hasta 7.12 veces la probabilidad de consumir alcohol durante el embarazo IC $95 \%$ [5.30, 9.56], seguido de la edad de inicio del uso de alcohol con 2.10 veces mayor probabilidad IC 95\% $[1.15,3.82]$ y haber tenido un embarazo previo $(O R=$ 1.87 IC $95 \%$ [1.03, 3.39].
En cuanto a los factores psicológicos que se relacionaron con el consumo de alcohol durante el embarazo (Tabla 3) se encontró que la depresión, ansiedad y menores niveles de emociones positivas, así como los niveles más elevados de aversión al riesgo de consumo, se relacionaron con el consumo (Salas-Wright et al., 2015, 2016). También se identificó que la ansiedad fue el factor que más aumenta el riesgo de beber alcohol durante el embarazo $(O R=3.48 / C 95 \%[2.97,6.80])$ seguido de la depresión $(O R=3.34$ IC 95\% [3.28, 5.26]) y el bajo nivel de aversión al riesgo ( $O R=0.56$ IC 95\% [0.43, 0.72]).

Dos de los estudios presentaron datos acerca de los factores sociales relacionados con el consumo de alcohol durante el embarazo en la adolescencia (Tabla 4). Las familias con un solo padre, tener amigos que fuman o que beben, y no tener pareja, se relacionó con el consumo de alcohol durante el embarazo (Vazquez-Nava et al., 2016; Veloso \& Monteiro, 2013). En cuanto a la probabilidad de riesgo, se identificó que no tener pareja aumenta en 2.82 veces (IC 95\% [1.62, 4.93]) el riesgo de consumir alcohol durante el embarazo, seguido de relacionarse con personas que fumen o beben con 2.11 (IC 95\% [1.25, 3.55]) más riesgo. Por último, en relación a los factores espirituales (Tabla 5), un estudio mostró que dar menos importancia a la religión se presentan como un factor relacionado con el consumo de alcohol durante el embarazo (Veloso \& Monteiro, 2013). Sin la presencia de este factor, se aumenta hasta 3.15 (IC95\% $[1.48,6.70])$ veces la probabilidad de consumir alcohol durante el embarazo.

\section{DISCUSIÓN}

En la presente revisión sistemática se encontraron cinco nuevos estudios a partir del 2011 que abordaron factores que se relacionan con el consumo de alcohol durante el embarazo en adolescentes. La mayoría de los estudios primarios incluidos en la presente revisión examinaron

Tabla 1

Prevalencias de consumo de alcohol en adolescentes embarazadas

\begin{tabular}{ll}
\hline Estudio & Prevalencia \\
\hline Veloso y Monteiro (2013) & $32.4 \%$ \\
Vazquez-Nava, Vazquez-R y Vazquez (2016) & $41.5 \%$ \\
Salas-Wrigth, Vaughn y Ugalde (2016) & $37.4 \%$ \\
Salas-Wright, Vaughn, Ugalde y Todic (2015) & $15.8 \%$ \\
\hline
\end{tabular}


Tabla 2

Factores personales relacionados con el consumo de alcohol en adolescentes embarazadas

\begin{tabular}{lcc}
\hline Variables & OR & $95 \%$ IC \\
\hline Veloso y Monteiro (2013) & 1.87 & {$[1.03,3.39]$} \\
Embarazo previo & 0.24 & {$[0.10,0.56]$} \\
Planificación del embarazo & 2.10 & {$[1.15,3.82]$} \\
Edad de inicio del uso de alcohol & & \\
Salas-Wright, Vaughn, Ugalde y Todic (2015) & & {$[5.30,9.56]$} \\
Edad entre 17 y 17 años & 0.48 & {$[0.30,0.80]$} \\
Segundo trimestre del embarazo & & {$[0.09,0.35]$} \\
\hline Tercer trimestre del embarazo & 0.18 & 0.12 \\
\hline
\end{tabular}

Nota: $O R=$ Frecuencias anormales (odds ratio); $I C=$ intervalos de confianza.

Tabla 3

Factores psicológicos relacionados con el consumo de alcohol en adolescentes embarazadas

\begin{tabular}{lcc}
\hline Variables & OR & $95 \%$ IC \\
\hline Salas-Wrigth, Vaughn y Ugalde (2016) & & \\
Bajo nivel de aversión al riesgo & 0.56 & {$[0.43,0.72]$} \\
Salas-Wright, Vaughn, Ugalde y Todic (2015) & 3.34 & {$[3.28,5.26]$} \\
Depresión & 3.48 & {$[2.97,6.80]$} \\
Ansiedad & 0.49 & {$[0.28,0.85]$} \\
Emociones positivas
\end{tabular}

Nota: $O R=$ Frecuencias anormales (odds ratio); $I C=$ intervalos de confianza. 
Tabla 4

Factores sociales relacionados con el consumo de alcohol en adolescentes embarazadas

\begin{tabular}{lrr}
\hline Variables & OR & $95 \%$ IC \\
\hline Vazquez-N, Vazquez-R y Vazquez (2016) & & \\
Familia con un solo padre & 1.79 & {$[1.17,2.74]$} \\
Tener amigos fumen o que toman & 2.11 & \\
Veloso y Monteiro (2013) & & \\
No tener pareja & $2.83,3.55]$ \\
\hline
\end{tabular}

Nota: $O R=$ Frecuencias anormales (odds ratio); $I C=$ intervalos de confianza .

Tabla 5

Factores espirituales relacionados con el consumo de alcohol en adolescentes embarazadas

\begin{tabular}{lcc}
\hline Variables & OR & $95 \%$ IC \\
\hline Veloso y Monteiro (2013) & 3.15 & {$[1.48,6.70]$} \\
\hline Importancia a la religión
\end{tabular}

Nota: $O R=$ Frecuencias anormales (odds ratio); $I C=$ intervalos de confianza.

los factores de tipo personal, de manera que el historial de consumo previo y la presencia de embarazos anteriores fueron los factores personales que se relacionaron significativamente con el consumo de alcohol durante el embarazo. Esto coincide con una revisión sistemática realizada por Bottorff et al. (2014) en adolescentes quienes mencionaban que aquellas usuarias de alcohol, tabaco o mariguana previo al embarazo, las que vivían por debajo de la línea de la pobreza y tenían embarazos previos también tenían más probabilidad de reportar consumo de alcohol durante el embarazo.

Asimismo, Skagerström et al., en una revisión en el 2011 en mujeres embarazadas concluyó que los embarazos y el consumo previo al embarazo eras los predictores más frecuentes del consumo de alcohol durante el embarazo. Por lo tanto, si las mujeres y sobre todo las adolescentes estuvieron expuestas a embarazos previos o si consumieron alguna sustancia antes de embarazarse, podrían en mayor medida usar alcohol durante dicha etapa.
Las revisiones sistemáticas que han examinado los factores psicológicos relacionados con el consumo de alcohol en mujeres embarazadas (Skagerström et al., 2011) y en adolescentes embarazadas (Bottorff et al., 2014) muestran que la depresión, la ansiedad y la baja autoestima son los factores psicológicos que más se relacionan con el consumo de alcohol durante el embarazo. En la presente revisión sistemática, entre los estudios recientes se localizaron estudios que identificaron la depresión y la ansiedad como los factores que más aumentaban la probabilidad de consumir alcohol en adolescentes embarazadas.

Entre los factores sociales, tener solo un padre, amigos que fuman y bajos niveles de calidez o control por parte de los padres también se relacionan con este consumo en indolentes. Al respecto, estudios como el de Bottorff et al. (2014) han mencionado que no tener un refuerzo positivo como apoyo social se relacionó con el consumo de alcohol durante el embarazo. Por lo tanto, 
la relación que se tiene con la familia, pareja y amigos se asocia con el uso de bebidas alcohólicas durante el embrazo adolescente. Por último, la presente revisión identificó que la importancia que se le brinda a la religión es un poderoso predictor del consumo de alcohol en embarazadas. Esto difiere de otras revisiones que no han encontrado relaciones significativas del aspecto espiritual religioso como un factor que se relaciona con el consumo (Veloso \& Monteiro, 2013). Se consideran limitaciones del presente trabajo en relación con el grupo de edad, debido a que dos de los estudios incluidos examinaron mujeres embarazadas de 20 años dentro de los grupos de edad estudiados. Se recomiendan futuras investigaciones que aborden factores protectores del consumo de alcohol para que se desarrollen intervenciones dirigidas a la prevención de este consumo en $\mathrm{AE}$.

\section{CONCLUSIONES}

Se confirma la prevalencia de consumo de alcohol durante el embarazo en adolescentes embarazadas y que existen factores presentes en las adolescentes embarazadas que incrementan la probabilidad de consumo de alcohol durante el embarazo hasta cuatro veces más. Los aspectos personales, como la presencia de embarazos previos y consumo de alcohol previo al embarazo, son los que más incrementan el número de veces la probabilidad de consumo durante el embarazo actual. Asimismo, los factores psicológicos como los sentimientos positivos, depresión y ansiedad incrementan la probabilidad de esta conducta. Tener un solo padre, amigos o pareja que fumen o beban y no tener pareja fueron los principales factores sociales identificados. Del mismo modo, se encontró que la importancia otorgada a la religión incrementa el riesgo de consumir alcohol durante el embarazo en la adolescencia. La presente investigación ha identificado estudios recientes en la temática en población adolescente; se espera que este estudio incremente la comprensión de los factores asociados al consumo de alcohol durante el embarazo y, posteriormente, sirva como referencia a fin de que las intervenciones fijen sus objetivos en adolescentes con menor edad, con embarazos previos y con un inicio temprano del uso de alcohol. También se recomienda mostrar especial atención en las adolescentes embarazadas con síntomas de depresión y ansiedad, debido a que incrementan el riesgo de consumo. Por último, la familia, amigos, así como la importancia de la religión también podrían formar parte importante para considerar en programas de intervención.

\section{FUENTES DE FINANCIAMIENTO}

No se recibió financiación para la realización de la presente revisión.

\section{DECLARACIÓN DE LOS AUTORES}

Los autores declaran no contar con conflicto de interés.

\section{AGRADECIMIENTOS}

A la Universidad Complutense de Madrid, por brindar los espacios de encuentro entre alumnos y profesores para poder realizar este trabajo en conjunto.

\section{REFERENCIAS}

Bottorff, J. L., Poole, N., Kelly, M. T., Greaves, L., Marcellus, L., \& Jung, M. (2014). Tobacco and alcohol use in the context of adolescent pregnancy and postpartum: A scoping review of the literature. Health and Social Care in the Community, 22(6), 561-574. doi: 10.1111/hsc. 12091

Lange, S., Probst, C., Heer, N., Roerecke, M., Rehm, J., Monteiro, M. G., Shield, K., de Oliveira, C., \& Popova, S. (2017). Actual and predicted prevalence of alcohol consumption during pregnancy in Latin America and the Caribbean: systematic literature review and meta-analysis TT - Prevalência real e prevista do consumo de álcool na gravidez na América Latina e Caribe: revis. Rev Panam Salud Publica, 41(1), e89-e89. http://www.scielosp.org/ scielo. php?script=sci_arttext\&pid=S1020-49892017000100301

Onwuka, C. I., Ugwu, E. O., Dim, C. C., Menuba, I. E., Iloghalu, E. I., \& Onwuka, C. I. (2016). Prevalence and predictors of alcohol consumption during pregnancy in South-Eastern Nigeria. Journal of Clinical and Diagnostic Research, 10(9), QC10-QC13. doi: 10.7860/JCDR/2016/21036.8449

Organización Mundial de la Salud (2019). Recomendaciones de la OMS sobre la prevención y la gestión del consumo del tabaco. 8. http://www.who.int/tobacco/

Popova, S., Lange, S., Probst, C., Parunashvili, N., \& Rehm, J. (2017). Prevalence of alcohol consumption during pregnancy and Fetal Alcohol Spectrum Disorders among the general and Aboriginal populations in Canada and the United States. European Journal of Medical Genetics, 6O(1), 32-48. doi: 10.16.1016/j.ejmg.2016.09.010

Ruisch, I. H., Dietrich, A., Glennon, J. C., Buitelaar, J. K., \& Hoekstra, P. J. (2017). Maternal substance use during pregnancy and offspring conduct problems: A meta-analysis. Neuroscience and Biobehavioral Reviews, 84(1), 325-336. doi: 10.1016/j. neubiorev.2017.08.014

Salas-Wright, C. P., Vaughn, M. G., \& Ugalde, J. (2016). A typology of substance use among pregnant teens in the United States. Matern Child Health J, 20(3), 646-654. doi: 10.1007/s10995-015-1864-1

Salas-Wright, C. P., Vaughn, M. G., Ugalde, J., \& Todic, J. (2015). Substance use and teen pregnancy in the United States: Evidence from the NSDUH 2002-2012. Addictive Behaviors, 45, 218225. doi: 10.1016/j.addbeh.2015.01.039

Skagerström, J., Alehagen, S., Häggström-Nordin, E., Årestedt, K., \& Nilsen, P. (2013). Prevalence of alcohol use before and during pregnancy and predictors of drinking during pregnancy: A 
cross sectional study in Sweden. BMC Public Health, 13(1). doi: 10.1186/1471-2458-13-780

Skagerström, J., Chang, G., \& Nilsen, P. (2011). Predictors of Drinking During Pregnancy A sistematic Review. Journal of Women's Health, 20(6), 901-913. doi: 10.1089/jwh.2010.2216

Vazquez-Nava, F., Vazquez-Rodríguez, C., Vazquez-Rodriguez,

E., Castillo-Ruiz, O., \& Iribar, M. (2016). Tobacco and alco- hol use in adolescents with unplanned pregnancies: relation with family structure, tobacco and alcohol use at home and by friends. African Health Science, 16(1), 27-35. doi: 10.4314/ ahs.v16i1.4

Veloso, L., \& Monteiro, C. (2013). Prevalencia y factores asociados al uso de alcohol en adolescentes embarazadas. Revista Latino-americana de Enfermagem, 21(1), 1-9 